\title{
A Novel Multi-layer Cooperative Decode and Forward Scheme
}

\author{
Reza Hoshyar, Fabien Héliot, and Rahim Tafazolli \\ Centre for Communication Systems Research (CCSR), Faculty of Electronics \& Physical Sciences, \\ University of Surrey, Guildford GU2 7XH, UK. Emails: \{F.Heliot, R.Hoshyar, R.Tafazolli\}@ surrey.ac.uk
}

\begin{abstract}
Decode-and-forward (DF) is one of the most popular approach to transmit information over a cooperative relay channel. In DF, information coming from different sources is simply combined regardless of their respective link qualities, and thus DF is not optimized for any propagation conditions. In this paper, we propose a novel transmit cooperation scheme that exploits asymmetric link qualities by using layered higher order modulation. A partial forwarding approach is followed by taking advantage of the layered structure: one part of the information is forwarded by a relay for increasing the robustness of the direct link and the rest of the information flows through the boosted direct link. Moreover, our scheme is based on a distributed serially concatenated encoding structure that can accommodate iterative decoding to further improve its performance. The complexity of our scheme is seemingly identical to DF scheme. Performance evaluation shows that our scheme provides a better spectral efficiency in asymmetric propagation conditions than DF scheme for both non-iterative and iterative decoding.
\end{abstract}

\section{INTRODUCTION}

Decode-and-forward (DF) is a well-known and documented technique that has been first designed for simple cooperative communications [1]-[3], and later it has been extended for multi-input-multi-output architectures [4]. In a simple cooperation scenario composed of a source (S) node, a destination (D) node, and a single relay ( $\mathrm{R}$ ) node, three node-to-node links are established, i.e., S-D, S-R, and R-D links. The common approach in DF over a cooperative relay channel is the full decoding of the source message followed by the forwarding of the whole message to the $\mathrm{D}$ node via the $\mathrm{R}$ node [5]. At the $\mathrm{D}$ node, the data which are received directly from the $\mathrm{S}$ node and indirectly through the $\mathrm{R}$ node are properly combined. However, this combining process is performed without taking into account the respective quality of the different links. Recently, it has been shown in [6] and [7] that additional knowledge as such can have a positive influence on the performance and spectral efficiency of DF scheme.

The transmission rate and format employed at $\mathrm{S}$ and $\mathrm{R}$ transmitters should be properly adjusted according to the expected quality of the links. Due to the broadcast nature of the $\mathrm{S}$ transmission targeted towards both the $\mathrm{R}$ and $\mathrm{D}$ nodes, the settings of modulation and coding format for this node face a dilemma in asymmetric link conditions. If the $\mathrm{R}$ node is sufficiently close to the $\mathrm{S}$ node and the $\mathrm{S}-\mathrm{R}$ link quality is high, then the $\mathrm{R}$ node can increase the communication reliability by helping the $\mathrm{S}$ node in its transmission process. This form of operation is commonly known as transmit (Tx) cooperation, and it can be used for throughput enhancement when the D node lies in shaded area or is close to the cellboundary, as advocated by the IEEE $802.16 \mathrm{~m}$ task group in [8]. In a typical $\mathrm{Tx}$ cooperation the S-R link capacity is much larger than the direct S-D link capacity. In this condition, an adjustment of the transmit format of $\mathrm{S}$ based solely on the minimum capacity of the two links leave the high capacity link unexploited. Here, we consider a different approach to the common DF scheme and create a layered transmission format in order to effectively and simultaneously exploit the different capacities of both S-D and S-R links, as we previously introduced in [7]. As a result, we propose a novel Tx cooperation scheme using layered higher order modulation (LHOM), refereed as DF-LHOM in the paper, that benefits from asymmetric propagation conditions. The created layered structure forms two parallel flows of information: one directly routed and one forwarded through the relay. The forwarded flow is further exploited as auxiliary information to create an effective robust constellation over the direct link. The auxiliary forwarded information of the layered modulation is error protected through an inner encoder that naturally drives us to adopt a serially concatenated encoding structure [9].

The rest of the paper is organized as follows. An overview of multi-layer modulation is given in Section II. In Section III, the system model of our novel DF-LHOM scheme is presented, and a detailed diagram illustrating both the LHOM and distributed serially concatenated structures is provided for each node. Simulation results of our novel DF-LHOM scheme for various LHOM constellations and iterative decoder settings are provided in Section IV. These results, which are compared against those obtained for DF scheme with iterative decoding (ID) or non-ID [5], show that our scheme performs better than these schemes in asymmetric propagation conditions. Furthermore, our DF-LHOM scheme is evaluated for various constellation labelings, and different puncturing patterns. Finally, conclusions are drawn in Section V.

\section{MUlti-LAYER MOdUlation}

In digital communications, a finite quadrature amplitude modulation (QAM) signal alphabet $\chi \subset \mathbb{C}$ associated with a one- to-one binary labeling map $\mu:\{0,1\}^{m} \mapsto \chi, m=\log _{2}|\chi|$, forms a constellation. The set $\chi$ can usually be defined as

$$
\chi \triangleq\left\{\begin{array}{l|l}
s_{i}=a+j b & \begin{array}{l}
a=-l+1+2[i \bmod (l)] \\
b=-l+1+2\lfloor i / l]\}
\end{array}
\end{array}\right\}
$$




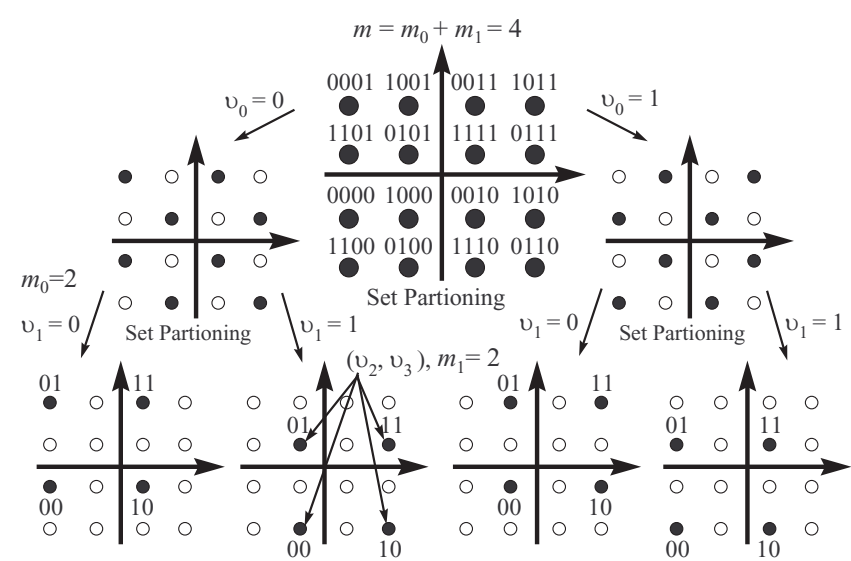

Fig. 1. 16-TLQAM $(2,2)$ modulation based on mixed-labeling.

for a rectangular $M$-QAM, where $s_{i}$ are the elements of $\chi$, $i \in\left[0,2^{m}-1\right]$, and $l=2^{\frac{m}{2}}$. A symbol error event may lead to different numbers of erroneous bits according to the labeling $\mu$ which is considered. Therefore, different types of labeling provide different bit-error rate (BER) performances. Among all the possible types, Gray and set partitioning (SP) based labelings have been widely used in radio communication systems. Gray labeling has been mainly used in bit interleaved coded modulation (BICM) systems, and a detailed definition of Gray labeling can be found in [10]. SP based labeling has been efficiently employed in [11] to directly improve Euclidean distance structure of jointly designed coded modulation systems. In general, any labeling map introduces some layer of unequal error protection on the input bits. Gray labeling attempts to reduce unequal protection to a minimum level while SP based labeling magnifies and utilizes this property. For instance in [12] and [13], SP has been applied to design a multi-level coded modulation system with different layers of protection and where each layer is coded separately. Furthermore, it has been shown in [14] that SP labeling provides better performance than Gray labeling in BICM with ID (BICM-ID). In this paper, we adopt a mixed strategy known as mixedlabeling, where SP is performed only for the first stages and then Gray labeling is used for each resulting sub-constellation. This strategy creates a multi-layer modulation, as illustrated in Fig. 1, where a 16-level two-layer QAM with two bits per layer, i.e., 16-TLQAM ${ }_{\left(m_{0}=2, m_{1}=2\right)}$, is presented.

We define

$$
\mathcal{L}=\left\{\sum_{k=0}^{m-1} u_{k} 2^{m-1-k} \mid u_{k} \in\{0,1\}\right\}
$$

as the set of decimal label of $\chi$. In the example of mixed-labeling illustrated in Fig. $1, \mathcal{L}=$ $\{12,4,14,6,0,8,2,10,13,5,15,7,1,9,3,11\}$ with respect to $\chi$ in (2). SP is applied for the first two stages resulting in four sub-constellations shown by black dots in Fig. 1. Each sub-constellation is an offset equivalent 4-QAM modulation with a minimum Euclidean distance reduces by a factor
1.115 compared to a genuine 4-QAM modulation. The first two label bits $\left(v_{0}, v_{1}\right)$ are used to select one of the sub-constellations and the last two label bits $\left(v_{2}, v_{3}\right)$, which are assigned by using Gray labeling, are used to select a point in the chosen sub-constellation. This structure creates two layered-streams composed of $m_{0}=2$ bits, i.e., $\left(v_{0}, v_{1}\right)$, and $m_{1}=2$ bits, i.e., $\left(v_{2}, v_{3}\right)$, respectively. It can be efficiently utilized in our novel DF-LHOM scheme. If bits $\left(v_{0}, v_{1}\right)$ are perfectly forwarded through the $\mathrm{R}$ node, then the $\mathrm{D}$ node would be aware of the selected sub-constellation and the uncertainty of the direct link, i.e., S-D link, would reduce to sub-constellation points. Effectively the source transmission would appear as an equivalent 4-QAM modulation for the S-D link and as a 16-QAM for the S-R link.

\section{SySTEM MODEL FOR THE DF-LHOM SCHEME}

Let us assume a simple cooperative communication system which is composed of three nodes, namely one S, one R, and one D node. A further assumption is that the nodes' transmission-reception is based on a simple protocol composed of two phases. The $\mathrm{S}$ node broadcasts its signal to the $\mathrm{R}$ and $\mathrm{D}$ nodes in the first phase of this protocol, and in the second phase only the $\mathrm{R}$ node transmits to the $\mathrm{D}$ node. Even though it would be more efficient to allow the $S$ and $R$ nodes to jointly transmit in the second phase as in [15], we adhere to this simple protocol for the convenience of introduction of the proposed approach. Phases I and II are composed of $N_{1}$, and $N_{2}$ symbol transmissions, respectively. In phase $\mathrm{I}$, the $\mathrm{S}$ node broadcasts signal sequence $\boldsymbol{x}_{1}=$ $\left(x_{1,0}, \ldots, x_{1, N_{1}-1}\right)$, and in phase II, the $\mathrm{R}$ node transmits the signal sequence $\boldsymbol{x}_{2}=\left(x_{2,0}, \ldots, x_{2, N_{2}-1}\right)$ to the D node, where $x_{i, j} \in \mathbb{C}$. Frequency-flat fading channels are assumed between any pair of transmitting-receiving nodes and channel state information of each link is assumed to be only known at each corresponding receiver node. The block diagram of our proposed DF-LHOM transmit cooperation scheme is depicted in Fig. 2. The exploited processing at the three nodes $\mathrm{S}, \mathrm{R}$, and D is illustrated in Fig. 2 (a), (b), and (c), respectively, and the generic structure of the assumed Forward Error Correction (FEC) encoder is detailed in Fig. 2 (d).

The source node transmitter relies on a FEC encoder $S$ to encode a sequence of bits $\boldsymbol{u}$ into a sequence of coded bits $\boldsymbol{c}=$ $\left(\boldsymbol{c}_{0}, \ldots, \boldsymbol{c}_{N_{1}-1}\right)$. It uses an $M$-TLQAM (mo $_{\left(m_{0}, m_{1}\right)}$ modulator to map the sequence $c$ to a vector of $N_{1}$ complex symbols $\boldsymbol{x}_{1}$, where $\boldsymbol{c}_{j}=\left(\boldsymbol{c}_{j}^{(0)}, \boldsymbol{c}_{j}^{(1)}\right)$ is an $m=\left(m_{0}+m_{1}\right)$-tuple of bits, with $\boldsymbol{c}_{j}^{(0)}=\left(v_{0}, v_{1}\right)$ and $\boldsymbol{c}_{j}^{(1)}=\left(v_{2}, v_{3}\right)$ for $M=16, m_{0}=2$, and $m_{1}=2$. Then, $\boldsymbol{x}_{1}$ is broadcasted at the same time towards the $\mathrm{R}$ and D nodes. We assume as in [8] that the S-R link is a high-reliability link, and that the S-D is a link with a far lower reliability than the S-R link. On the one hand, the high-quality of the S-R link implies that the $\mathrm{R}$ node is able to reliably decode the two bit sub-sequences $\boldsymbol{c}^{(0)}$ and $\boldsymbol{c}^{(1)}$, which are conveyed through the $M$-TLQAM $\left(m_{0}, m_{1}\right)$ and represent the two layers of information. On the other hand, the low-quality of the S-D implies that the D node is able to decode the lowerlayer of information $\boldsymbol{c}^{(1)}$ provided that in the mean time the 


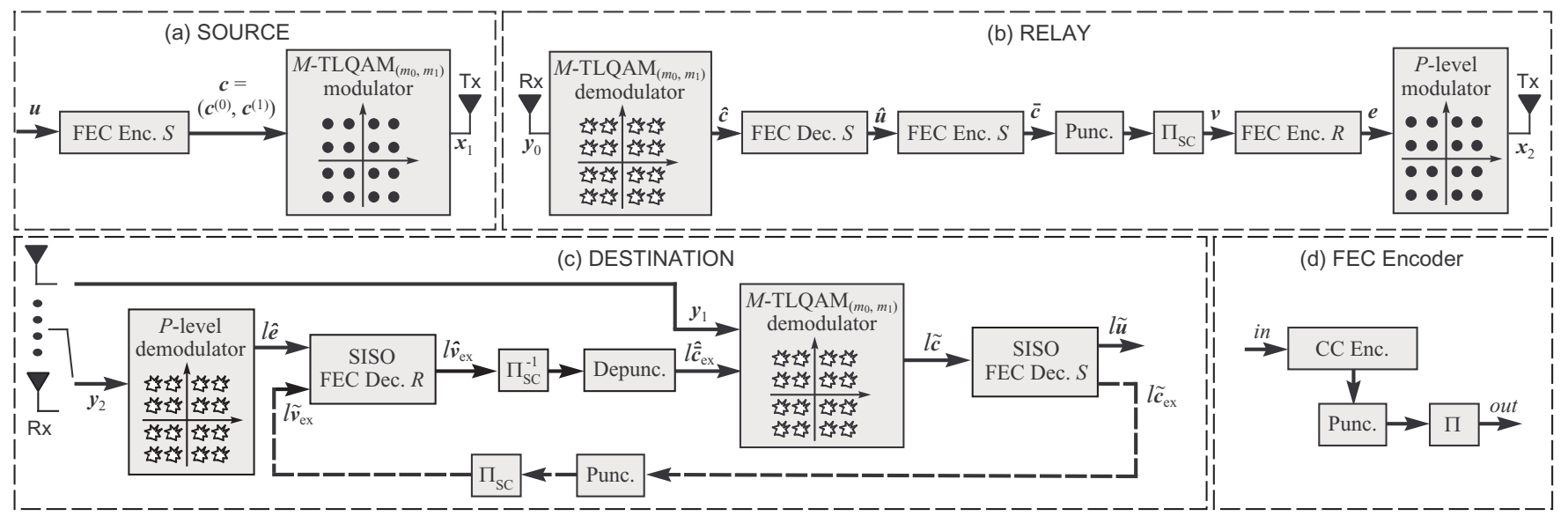

Fig. 2. : Block diagram of the proposed DF-LHOM scheme: (a) the source node transmitter, (b) the relay node receiver/transmitter, (c) the destination node receiver, and (d) the FEC encoder generic structure.

upper-layer $\boldsymbol{c}^{(0)}$ has been somehow received with sufficient reliability. In other words, by using the layered modulation structure, the sequence $\boldsymbol{c}$ is partially forwarded by the R node, i.e., only the $c^{(0)}$ part, and $c^{(0)}$ is used as auxiliary information at the D node to decode $c^{(1)}$.

At the receiver side of the R node, $\boldsymbol{x}_{1}$ is received as $\boldsymbol{y}_{0}$, demodulated as $\widehat{\boldsymbol{c}}$ and decoded as $\widehat{\boldsymbol{u}}$. Following this step, $\widehat{\boldsymbol{u}}$ is re-encoded into $\overline{\boldsymbol{c}}$ by using the same FEC encoder $S$ that has been used at the source. Our scheme requires only $\overline{\boldsymbol{c}}^{(0)}$ to be forwarded, therefore $\overline{\boldsymbol{c}}^{(1)}$ can be either fully punctured, partially punctured, or even compressed as in [7]. The stream of punctured coded-bits is then interleaved by using $\Pi_{\mathrm{SC}}$, encoded via the FEC encoder $R$ to protect $v$, and $\boldsymbol{e}$ is finally mapped into a vector of symbols $\boldsymbol{x}_{2}$ by using a $P$ level modulation, e.g., QAM, and transmitted towards the D node. Notice that the levels of modulation $M$ and $P$ can be adjusted independently. This makes our scheme fairly flexible in terms of data rate, which can be adapted in function of the propagation conditions. In comparison with DF, an additional FEC encoder $S$ is required at the $\mathrm{R}$ node, which increases slightly its complexity. However, it forms with the interleaver $\Pi_{\mathrm{SC}}$ and the second FEC encoder $R$ a serially concatenated encoding structure. This structure can be used to improve the performance through ID at the receiver side [9].

At the receiver side of the $\mathrm{D}$ node, the signal received from the R node, $\boldsymbol{y}_{2}$, is demodulated as $l \widehat{\boldsymbol{e}}$, and decoded via the Soft-Input Soft-Output (SISO) FEC decoder $R$ as $l \widehat{\boldsymbol{v}}_{\mathrm{ex}}$, where $l \widehat{\boldsymbol{e}}$ and $l \widehat{\boldsymbol{v}}_{\mathrm{ex}}$ are Log Likelihood Ratio (LLR) values. Afterwards, $l \widehat{\boldsymbol{v}}_{\mathrm{ex}}$ is de-punctured and de-interleaved as $l \widehat{\overline{\boldsymbol{c}}}_{\mathrm{ex}}$ that contains the full upper-layer and the punctured lower-layer of information. This stream is used as extrinsic information in order to softly demodulate and de-map the stream $\boldsymbol{y}_{1}$ coming from the $\mathrm{S}$ node. The aggregated stream of LLR values $l \widetilde{\boldsymbol{c}}$ is finally decoded via a SISO FEC decoder $S$, and the output LLR stream $l \widetilde{\boldsymbol{u}}$ corresponding to the output bit sequence $\widetilde{\boldsymbol{u}}$ is obtained. In addition, the LLR values of $l \widetilde{\boldsymbol{u}}$ can be further refined by using ID. As a result of the serially concatenated encoding structure implemented at the relay, the $\mathrm{D}$ node is equivalent to a distributed serially concatenated iterative decoder. At the end of the first iteration previously described, the coded LLRs $l \widetilde{\boldsymbol{c}}_{\mathrm{ex}}$, which are provided by the SISO FEC decoder $S$, are punctured and interleaved as $l \widetilde{\boldsymbol{v}}_{\mathrm{ex}}$ and are then fed back into the SISO FEC decoder $R$ to perform the ID process. The complexity of this node is seemingly identical to its DF scheme counterpart. Notice that in contrast to the conventional serial concatenation decoder, the considered iterative decoder is composed of three SISO components, i.e., two for the FEC decoders $S$ and $R$, and one for the $M$-TLQAM $\left(m_{0}, m_{1}\right)$ demodulator.

\section{Performance Analysis}

In our simulations, we assume a single-tap Rayleigh fast fading channel with a normalized Doppler frequency of 100 $\mathrm{Hz}$ between the various links, S-D, S-R, and R-D. The FEC encoders $S$ and $R$ are half-rate (7,5)-CC with zero padding. All the nodes have a single transmit/receive antenna. We define $\Delta_{\text {S-R }}$ and $\Delta_{\text {R-D }}$ as the SNR offsets of the S-R and R-D links, respectively, according to the S-D link. We assume that the S-R link is highly reliable compare to the S-D link and set $\Delta_{\mathrm{S}-\mathrm{R}} \geq 10 \mathrm{~dB}$. We also define $\gamma_{D F}$ and $\gamma_{D F-L H O M}$ as the SNRs require by the DF and DF-LHOM schemes, respectively, to achieve a given BER for fixed $\Delta_{\text {S-R }}$ and $\Delta_{R-D}$ values. The difference in $\mathrm{dB}$ between these two SNRs, i.e., $G \triangleq\left(\gamma_{D F-L H O M}(\mathrm{~dB})-\gamma_{D F}(\mathrm{~dB})\right)$, provides a measure of the SNR gain that our scheme can achieve in comparison with the conventional DF scheme [5]. Three types of labeling have been employed at the $\mathrm{S}$ node, Gray, i.e., $m_{0}=0, m_{1}=m$, mixed, i.e., $m_{0}=m / 2, m_{1}=m / 2$, and full SP (FSP), i.e., $m_{0}=m$, $m_{1}=0$. For the case of $M=16$, their respective decimal label sets can be expressed as

$$
\begin{aligned}
& \mathcal{L}_{\text {Gray } 16}=\{2,6,14,10,3,7,15,11,1,5,13,9,0,4,12,8\}, \\
& \mathcal{L}_{\text {mixed16 }}=\{0,4,2,6,8,12,10,14,1,5,3,7,9,13,11,15\}, \\
& \mathcal{L}_{\text {FSP16 }}=\{14,6,13,5,2,10,1,9,12,4,15,7,0,8,3,11\},
\end{aligned}
$$




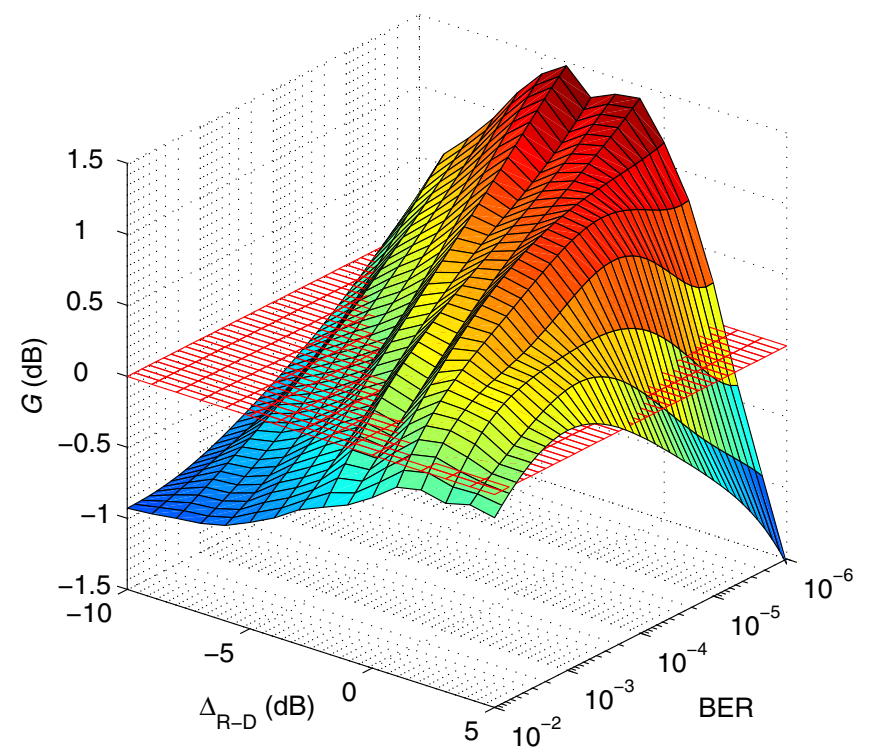

Fig. 3. SNR gain of our DF-LHOM scheme compared to DF scheme functions of $\Delta_{\mathrm{R}-\mathrm{D}}$ and the BER, $\Delta_{\mathrm{S}-\mathrm{R}}=10 \mathrm{~dB}, M=16, P=16$.

according to $\chi$ in (2). For the case of $M=256$, the label set $\mathcal{L}_{\text {mixed256 }}$ is available at the end of the paper. Various puncturing patterns $\mathrm{P}_{i}$ have been defined according to the modulation based on mixed-labeling. If $\mathrm{P}_{i}$ is applied, then $i$ bits among the $m_{1}$ bits of $\overline{\boldsymbol{c}}_{j}^{(1)}$ for each symbol $\overline{\boldsymbol{c}}_{j}$ are punctured. For instance, $\mathrm{P}_{0}$ means that no bits are punctured and $\mathrm{P}_{m_{1}}$ that $\overline{\boldsymbol{c}}^{(1)}$ is fully punctured. A P-QAM modulator with Gray labeling has been used at the $\mathrm{R}$ node. The aggregated performance of our scheme, i.e., $\boldsymbol{u}$ vs. $\widetilde{\boldsymbol{u}}$ is evaluated for ID or non-ID. The performances of DF-LHOM and DF schemes are compared for the same data rate.

In Figs. 3 and 4 , the SNR gain $G$ functions of $\Delta_{\text {R-D }}$ and the BER is displayed for $M=16, m_{0}=2, m_{1}=2, \Delta_{\mathrm{S}-\mathrm{R}}=10$ $\mathrm{dB}$ and $M=256, m_{0}=4, m_{1}=4, \Delta_{\mathrm{S}-\mathrm{R}}=15 \mathrm{~dB}$, respectively. Moreover, $P=16$, a full puncturing of $\overline{\boldsymbol{c}}^{(1)}$, and non-ID are assumed. First, the results show that our DFLHOM scheme achieves a gain compare to DF scheme in between 0.5 to $1.5 \mathrm{~dB}$ at BER of $10^{-5}$ for $\Delta_{\mathrm{R}-\mathrm{D}} \in[-8,3]$ in the case of $M=16$, and a gain in between 0.5 to 3 $\mathrm{dB}$ at BER of $10^{-6}$ for $\Delta_{\mathrm{R}-\mathrm{D}} \in[-20,0]$ in the case of $M=256$. Intuitively, the BER provides a measure of the reliability of the S-D link, since higher SNRs of the S-D link are required to achieve a lower BER. We observe that for high BERs, i.e., a low quality of the S-D link, and low $\Delta_{\text {R-D }}$ values, our scheme performs worst than DF scheme. In this configuration, the reliability of $\overline{\boldsymbol{c}}^{(0)}$, which has been forwarded by the relay over the R-D link, is low and thus the inaccuracy in the sub-constellation selection implies a sharp performance degradation. As the BER decreases, the quality of both the S-D and R-D links increases, then the subconstellation selection becomes more reliable, and our DFLHOM scheme outperforms DF scheme. This result indicates that the pre-selection of information is more efficient than the simple combination in asymmetric link quality conditions.

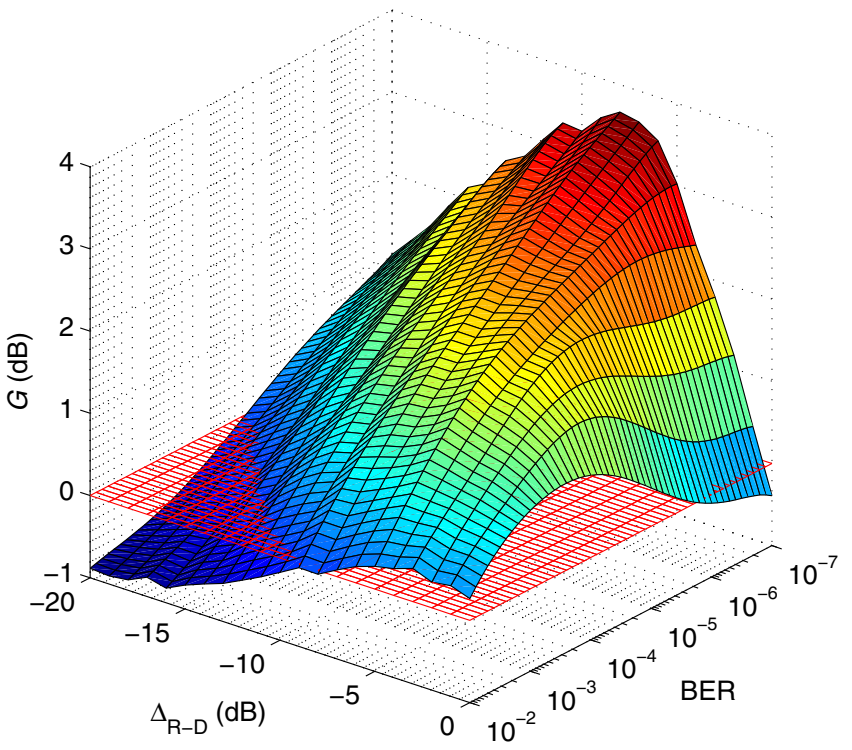

Fig. 4. SNR gain of our DF-LHOM scheme compared to DF scheme functions of $\Delta_{\mathrm{R}-\mathrm{D}}$ and the BER, $\Delta_{\mathrm{S}-\mathrm{R}}=15 \mathrm{~dB}, M=256, P=16$.

Moreover, as depicted in Figs. 3 and 4, our DF-LHOM scheme does not provide a constant gain for any $\Delta_{\mathrm{R}-\mathrm{D}}$ values, $G$ drops sharply for very low BERs, i.e., high quality of the S-D link, and $\Delta_{\text {R-D }}>0$. In this case, the equivalent S-R-D link is highly reliable, then $\overline{\boldsymbol{c}}^{(0)}$ is highly reliable and the sub-constellation selection is highly reliable. However, $\overline{\boldsymbol{c}}^{(1)}$, which has been punctured at the relay, is not available at the destination for refining $c^{(1)}$ that has been transmitted over the S-D link. Thus, our scheme misses $\overline{\boldsymbol{c}}^{(1)}$ at the D node to achieve the same diversity and performance as DF scheme.

In Fig. 5, we compare the DF-LHOM and DF PER performances for $\Delta_{\mathrm{S}-\mathrm{R}}=15 \mathrm{~dB}, \Delta_{\mathrm{R}-\mathrm{D}}=-10 \mathrm{~dB}, M=256$, $m_{0}=4, m_{1}=4, P=16$, a full puncturing of $\overline{\boldsymbol{c}}^{(1)}$, and ID, i.e., 1,2 and 10 iterations (it.). Notice that we have used the distributed parallel concatenation turbo decoder presented in [5] to obtain the results for DF scheme. The result shows that our DF-LHOM scheme outperforms DF scheme for any number of iterations. However, the performance difference between the two schemes is lower for 10 iterations than for a single one, i.e., $0.7 \mathrm{~dB}$ instead of $1.2 \mathrm{~dB}$ at a PER of $10^{-3}$. It indicates that the distributed parallel concatenation turbo decoder of DF scheme is able to harvest more gain from the ID process than the serial concatenation structure of our decoder, but the multi-layer structure of our scheme is still more efficient than the non-layer one used in DF.

In Fig. 6, we analyze the performance of the DF-LHOM scheme for different labelings, various puncturing patterns, $\Delta_{\mathrm{S}-\mathrm{R}}=15 \mathrm{~dB}, \Delta_{\mathrm{R}-\mathrm{D}}=-10 \mathrm{~dB}, M=256, P=16$, and 10 iterations of the decoder. Notice that here we employ the same puncturing pattern $\mathrm{P}_{4}$ defined according to mixed labeling for every other labelings. The first three curves show that our proposed mixed-labeling is the most efficient labeling in order to get the most gain out of the multi-layer modulation structure, since it outperforms the Gray and FSP labelings by 


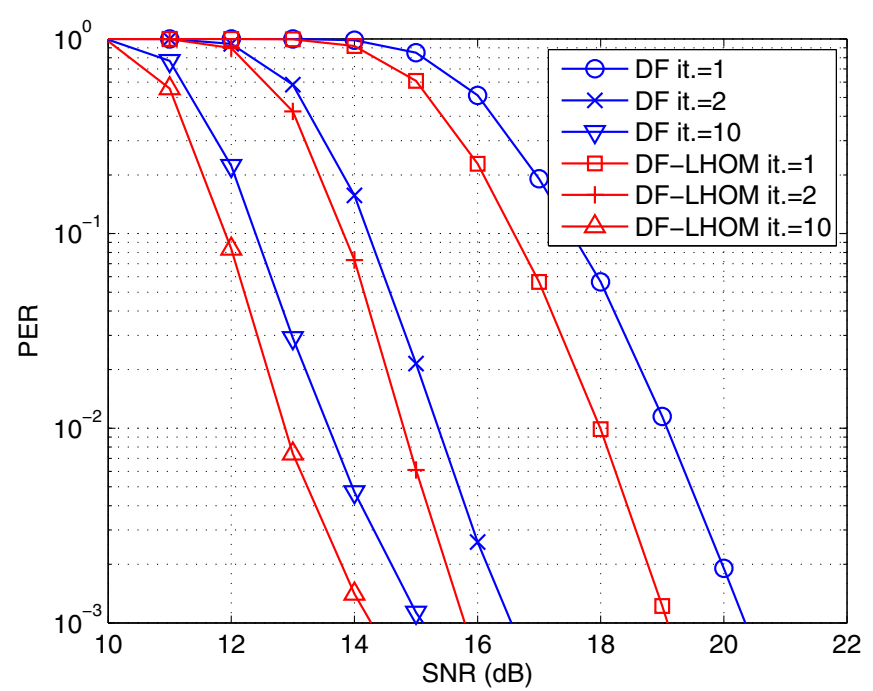

Fig. 5. PER comparison of the DF-LHOM against DF scheme for $\Delta_{\mathrm{S}-\mathrm{R}}=15$ $\mathrm{dB}, \Delta_{\mathrm{R}-\mathrm{D}}=-10 \mathrm{~dB}, M=256, P=16$, and various numbers of decoding iterations.

9 and $2 \mathrm{~dB}$, respectively, at a PER of $10^{-3}$. These results are in line with the ones obtained in [14] for BICM-ID. Moreover, it can be observed by comparing the first, fourth, fifth and sixth curves that increases the number of punctured bits decreases the performance. However, the data rate of the second transmission phase can be double, i.e., using $\mathrm{P}_{4}$ instead of $\mathrm{P}_{0}$, by increasing the SNR of only $2 \mathrm{~dB}$ at a PER of $10^{-2}$.

\section{CONCLUSION}

A novel transmit cooperation scheme that benefits from asymmetric propagation conditions has been designed in this paper. A different approach to DF scheme has been followed to create a layered transmission format by using mixed-labeling in order to effectively exploit the capacity of both S-D and S-R links. In addition to the layered architecture, a distributed serially concatenated encoding structure has been designed to further improve the performance of our scheme. Several mixed-labeling patterns for different levels of modulation have been introduced. Performance analysis has shown that mixedlabeling is efficient to take advantage of the asymmetric nature of the broadcast phase via the multi-layer modulation and to effectively exploit the ID through the distributed serially concatenated structure. As a result, this scheme is spectrally more efficient than DF scheme in asymmetric conditions. This paper presents promising preliminary results for our DF-LHOM scheme. In the future, these results will be complemented by a thorough theoretical analysis.

\section{ACKNOWLEDGMENT}

This work has been performed in the framework of the FP7 project ROCKET IST-215282 STP, which is funded by the European Community.

\section{REFERENCES}

[1] A. Sendonaris, E. Erkip, and B. Aazhang, "User cooperation diversity part i - system description," IEEE Trans. Commun., vol. 51, no. 11, pp. 1927-1938, Nov. 2003.

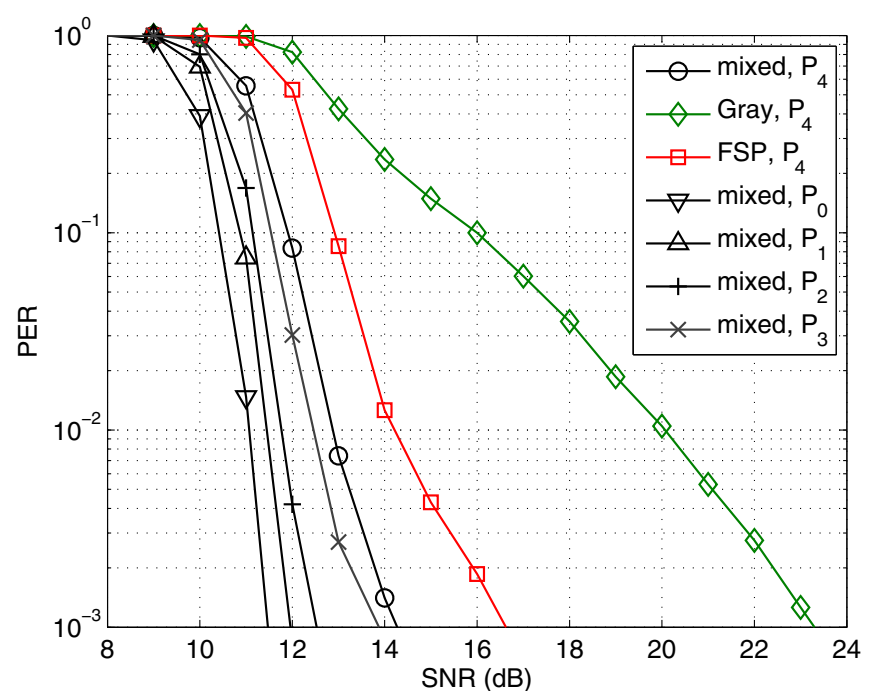

Fig. 6. PER performance of the DF-LHOM scheme for different labelings and puncturing patterns, $\Delta_{\mathrm{S}-\mathrm{R}}=15 \mathrm{~dB}, \Delta_{\mathrm{R}-\mathrm{D}}=-10 \mathrm{~dB}, M=256, P=16$, and 10 iterations.

[2] — - "User cooperation diversity part ii - implementation aspects and performance analysis," IEEE Trans. Commun., vol. 51, no. 11, pp. 1939 1948, Nov. 2003.

[3] J. N. Laneman, D. N. C. Tse, and G. W. Wornell, "Cooperative diversity in wireless networks: Efficient protocols and outage behavior," IEEE Trans. Inform. Theory, vol. 50, no. 12, pp. 3062-3080, Dec. 2004.

[4] M. Janani, A. Heday, T. Hunter, and A. Nosratinia, "Coded cooperation in wireless communications: space-time transmission and iterative decoding," IEEE Trans. Signal Processing, vol. 52, no. 2, pp. 362-371, Feb. 2004.

[5] flexible relay wireless OFDM-based networks (FIREWORKS) consortium, "Preliminary description of the phy techniques for relay/mesh based networks," IST Project FP6-027675, Tech. Rep. 4D1, Nov. 2006. [Online]. Available: http://fireworks.intranet.gr/fireworks_docspublic/ Fireworks_4D1.pdf

[6] R. Hoshyar and R. Tafazolli, "Soft decode and forward of mqam modulations for cooperative relay channels," in Proc. VTC Spring 2008, Marina Bay, Singapore, May 2008.

[7] R. Hoshyar, F. Héliot, and R. Tafazolli, "A low-complexity transmit cooperation scheme based on layered higher order modulation," in Proc. ICT Mobile Summit 2008, Stockholm, Sweden, 2008.

[8] ieee 802.16 broadband wireless acess working group, "Ieee 802.16 system requirements," IEEE, Tech. Rep. IEEE 802.16m-07/002r4, Oct. 2007. [Online]. Available: http://wirelessman.org/tgm/docs/80216m-07_ 002r4.pdf

[9] S. Benedetto and G. Montorsi, "Serial concatenation of interleaved codes: Performance analysis, design, and iterative decoding," IEEE Trans. Inform. Theory, vol. 44, no. 3, pp. 909-926, May 1998.

[10] G. Caire, G. Taricco, and E. Biglieri, "Bit-interleaved coded modulation," IEEE Trans. Inform. Theory, vol. 44, no. 3, pp. 927-946, May 1998.

[11] G. Ungerboeck, "Channel coding with multilevel/phase signals," IEEE Trans. Inform. Theory, vol. 28, no. 1, pp. 55-67, Jan. 1982.

[12] R. H. Morelos-Zaragoza, M. P. C. Fossorier, S. Lin, and H. Imai, "Multilevel coded modulation for unequal error protection and multistage decoding .i. symmetric constellations," IEEE Trans. Commun., vol. 48, no. 2, pp. 204-213, Feb. 2000.

[13] H. Imai and S. Hirakawa, "A new multilevel coding method using errorcorrecting codes," IEEE Trans. Inform. Theory, vol. 23, no. 3, pp. 371377, May 1977.

[14] L. Xiaodong and J. Ritcey, "Bit-interleaved coded modulation with iterative decoding," IEEE Commun. Lett., vol. 1, no. 6, pp. 169-171, Nov. 1997.

[15] flexible relay wireless OFDM-based networks (FIREWORKS) consortium, "Detailed description of aas and selected phy techniques for relay/mesh based networks," IST Project FP6- 
$\mathcal{L}_{\text {mixed256 }}=\{34,98,226,162,38,102,230,166,46,110,238,174,42,106,234,170,50,114,242,178,54,118,246,182,62,126$, $254,190,58,122,250,186,18,82,210,146,22,86,214,150,30,94,222,158,26,90,218,154,2,66,194,130,6,70,198,134,14$, $78,206,142,10,74,202,138,35,99,227,163,39,103,231,167,47,111,239,175,43,107,235,171,51,115,243,179,55,119$, $247,183,63,127,255,191,59,123,251,187,19,83,211,147,23,87,215,151,31,95,223,159,27,91,219,155,3,67,195,131$, $7,71,199,135,15,79,207,143,11,75,203,139,33,97,225,161,37,101,229,165,45,109,237,173,41,105,233,169,49,113$, $241,177,53,117,245,181,61,125,253,189,57,121,249,185,17,81,209,145,21,85,213,149,29,93,221,157,25,89,217$, $153,1,65,193,129,5,69,197,133,13,77,205,141,9,73,201,137,32,96,224,160,36,100,228,164,44,108,236,172,40$, $104,232,168,48,112,240,176,52,, 116,244,180,60,124,252,188,56,120,248,184,16,80,208,144,20,84,212,148,28$, $92,220,156,24,88,216,152,0,64,192,128,4,68,196,132,12,76,204,140,8,72,200,136\}$.

027675, Tech. Rep. 4D2, Sept. 2007. [Online]. Available: http://fireworks.intranet.gr/fireworks_docspublic/Fireworks_4D2.pdf 